\title{
The effects of Fresnel Zone in communication theory based on radio waves
}

\author{
Javad AHMADI \\ Electrical engineering graduate - Expert RF circuits and radio links
}

\begin{abstract}
Summary
As the visible light absorption of radio waves also have the ability to be absorbed. Radio waves are also based on the principle of focusing obey the laws of physics. Or the phase shift of focus in telecommunications is an important factor for Radio Communications, which includes discussion is lengthy. (Refer to the modulation of radio communications) Several factors such as reflection, height, type and frequency transceivers are involved in the creation of Fresnel zone.
\end{abstract}

Keywords: Fresnel zone - an area radio wave radiation - radio waves casualties

\section{Introduction}

According to the theory of radio-based communication has to be analyzed in this context. One of these theories is the theory of Fresnel Zone by which we can achieve maximum efficiency of a radio link. This theory is based on line of sight and range of the transmitter and receiver to the level necessary for the proper functioning of our show. Given my experience in practical projects and recognize the importance of radio communication outlines the various aspects discussed Fresnel Zone. In the primary sector will be discussed on pages Fresnel Zone Fresnel Zone is introduced, followed by three-dimensional environment to further clarify the sensitivity of the issue for specialists to be cherished. It is hoped that special attention and effort in applying the principles of radio communication technology faster than the walk to the summit.

\section{Fresnel Plate Zone - Fresnel zone plates}

In the figure below, showing a plate waves (front view) are to be discussed valuation of pages in the Fresnel Zone. [3] 


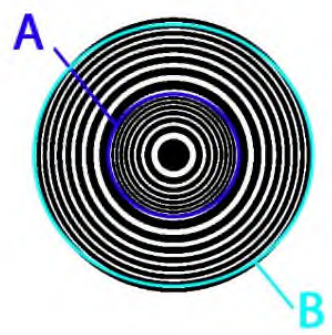

A- The point is that the focus of the transmission or the Fresnel Zone is the most important medium of transmission has the greatest impact on the quality of transmission. Be concentrated in the central region of Zone 2 is far more.

B- this environment based on proportionality diameter (Diameter) and beam angle (Beam) antennas, a link is created. No matter which direction we go out of the Fresnel Zone will be worth less waves.

Fresnel Zone radius is reduced by increasing the width of the waves. This means that if the disproportionate waves to the transmitter and receiver are not properly send and receive. [1]

\section{Fresnel Zone - Fresnel zone and how to recognize it}

If we look at the image below as side there will be a connection between two points.

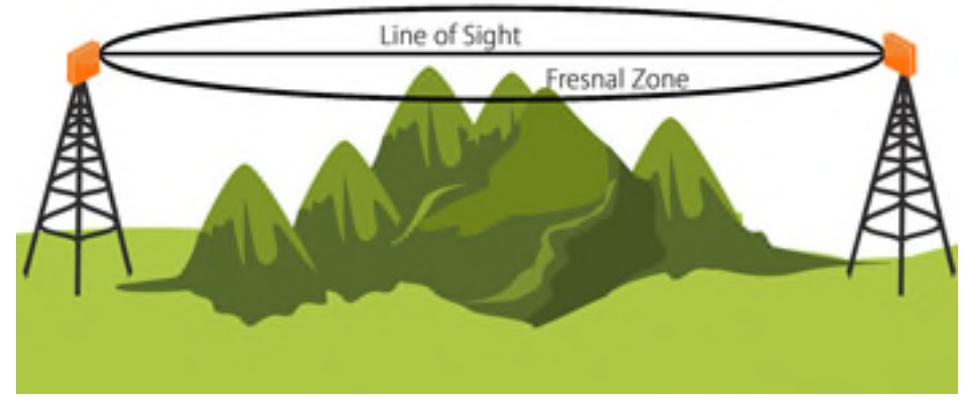

Groups oval Fresnel Zone indicator will be the basis for a line of sight, which is based on a direct line of sight (Line Of Sight) radio equipment requires a line of sight between the RF .artbat call is. Two types of line of sight must be considered: the visual line of sight and line of sight radio frequency.

The transmitter and receiver are able to see each other's visual line of sight. To be clear, unobstructed view to be successful wireless link between the transmitter and receiver to be there. Unobstructed line of sight, which means that few obstacles in the way of RF signals between devices or there is no obstacle. 
Radio frequency and unobstructed line of sight between the receiver and radio transmitter is a straight line. The radio frequency transmission line by the area of the so-called Fresnel Zone is surrounded.

If Fresnel total area of more than $40 \%$ blockage, the line of sight RF or radio transmission between a transmitter and a receiver could be affected and weakened. These blockages can result from a variety of sources such as trees, buildings, terrain or other obstacles such as the curvature of the Earth.

One way of thinking about the line of sight to each other by comparing two people that look. If two people have the same stature that far apart standing, maintain eye contact, have a good visual line of sight. Besides direct vision, peripheral vision as well. This peripheral vision gives people the ability to move objects out of direct line of sight or peripheral vision. This view direct eye contact with Fresnel Zone is a side view similar to that theory.

How the obstruction Fresnel Zone

One of the ways the embodiment of Fresnel Zone is that your eyes focused on the region on a wall and make sure that the left and right sides of your vision or mobility barriers exist. Keep your hands in Trfyntan down. Focus your eyes for a minute or so to continue. Then the left hand, right hand, or both intended and while you see around you blocked them side by side to raise their heads. You will notice that once you get your hands closer you get to hug your head, see things or move from side to side will be blocked by your hands. This is an example of a Fresnel zone is blocked.

Note: $60 \%$ of the total Fresnel zone should be free of obstacles RF line of sight is restored. [5]

This area has already been facing see also analyzed. Given the above explanations and calculations relations will be discussed Fresnel Zone.

\section{Reflect on Fresnel Zone}

The radio-based communication efficiency may decline in the rain it might have after hours after the rain continued. Something which links to the sea surface and sea-to-sea well as the challenges are familiar. The challenge presented by radiation and reflected waves to surfaces that reflect visible and invisible waves. This will defeat waves, resulting in erosion of the transmission waves. Perhaps one of the most important reasons for insisting on the installation of equipment at the highest elevation of this issue. Although outside the scope of Fresnel Zone has far less power, but in the case of reflection, greatly affect the overall performance of a radio link. In the screenshot below. 


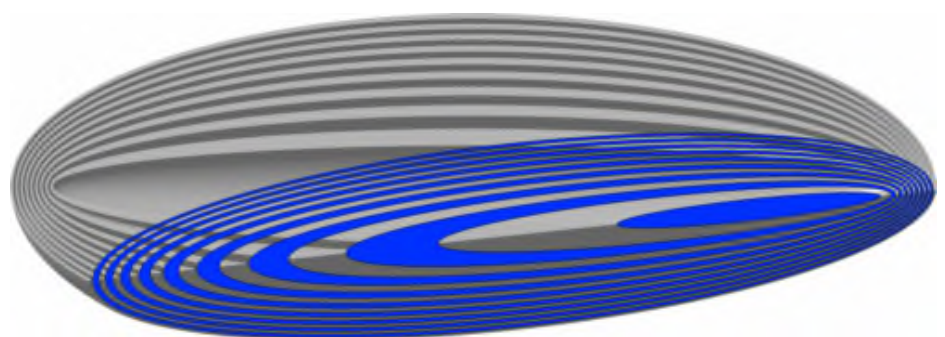

The main wave of gray in the image above marked the reflection of outer slopes generally been a diversion waves. This sometimes causes a sharp drop in performance, and in some cases entirely cut off radio contact will take. [2]

The best strategies to deal with these mounting challenges at several-fold, relative to the radius of the Fresnel Zone is free.

\section{Computing Fresnel Zone}

Through the following equation can be calculated from the height required.

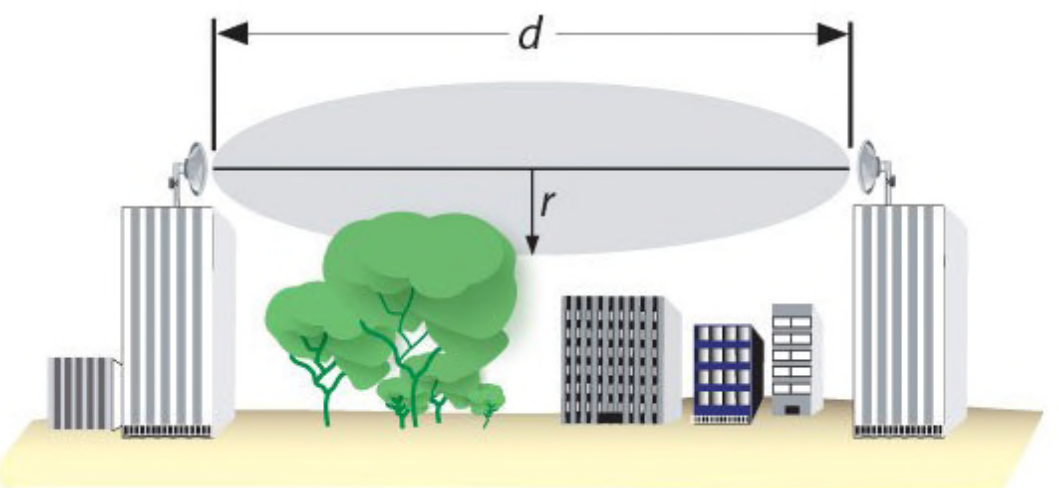

$(\mathrm{R}=17.32 * \operatorname{sqrt}(\mathrm{N}(\mathrm{d} 1 * \mathrm{~d} 2)) /(\mathrm{f} * \mathrm{~d})$

$\mathrm{r}=$ radius of the signal to $\mathrm{m}$

$\mathrm{n}=$ area calculation

$\mathrm{d} 1, \mathrm{~d} 2$ = distance to each of the radio barrier to $\mathrm{m}$

$\mathrm{d}=$ all link distance in meters

$\mathrm{f}=$ frequency in $\mathrm{MHz}$

or

$(\mathrm{R}=8.657 * \operatorname{sqrt}(\mathrm{D} / \mathrm{f}))$

$\mathrm{D}=$ total distance in kilometers

$\mathrm{f}=$ frequency in $\mathrm{GHz}$

among which use a $100 \%$ clean and noise free area will need to be able to fully benefit from the actual equipment. 
* Hogan principle: rather than particle theory that space, but is built and everything is always so in vibration wave and did not calm

It is also possible to obtain the radius of the Fresnel Zone will use the following formula:

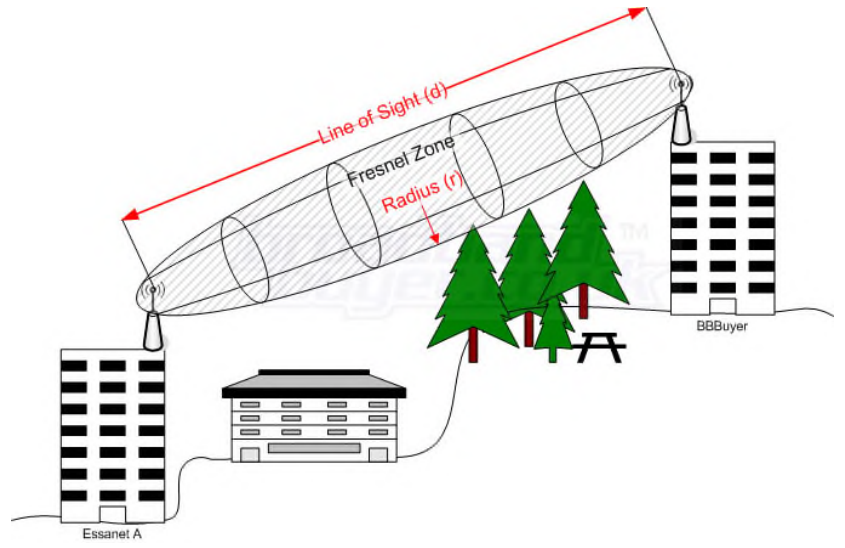

$F_{n}=\sqrt{\frac{n \lambda d_{1} d_{2}}{d_{2}+d_{2}}}$

d1 distance from the midpoint of the transmitting antenna

$\mathrm{d} 2$ are antenna from the midpoint

$\mathrm{F}$ frequency in $\mathrm{GHz}$

Calculation of land Qvs

barriers or obstacles we collect

It is calculated by the following equation

$\mathrm{H}=1000 * \mathrm{D} 2 / 8 \mathrm{Er}$

$\mathrm{H}=$ height of the arch in the middle ground between two points

$\mathrm{D}=$ total distance in kilometers

$\mathrm{Er}=$ effective radius of the Earth in terms of $\mathrm{km}$ which $8504 \mathrm{~km}$ are considered

\section{6-Conclusion}

According to material presented can be downloaded at the end of the notice of the environment, and telecommunications equipment makes possible the lowest cost, most radio waves to transmit data acquired. While projects without careful calculation, with the expenditure side face highfrequency including the purchase of equipment. Equipment that operate with high power, noise and pollution, but the ultimate benefit will be lower than expected. 
Bulletin de la Société Royale des Sciences de Liège, Vol. 85, 2016, p. 729 - 734

\section{References}

1. A.J. Fresnel, "Calcul de l'intensité de la lumière au centre de l'ombre d'un écran et d'une ouverture circulaires éclairés par un point radieux", in: Euvres Complètes d'Augustin Fresnel, Imprimerie Impériale, Paris, 1866

2. VDI / VDE 5575 Blatt 6: 2009-03 Röntgenoptische Systeme, Reflexionszonenplatten (Xray optical systems; Reflexion zone plates), Berlin, Beuth Verlag, 2009

3. Jump up ^ "Zone Plates". X-Ray Data BookleCenter for X-ray Optics and Advanced Light Source, Lawrence Berkeley National Laboratory. Retrieved 13 January 2015.

4. 4-Jump up ^ Joseph W. Goodman (2005). Introduction to Fourier Optics ( $3^{\text {rd }}$ ed.). p. 125. ISBN 0-9747077-2

5. 5. Wireless Communication Specialist - Technical Complex Tehran, Press Dibagaran - Mr. Sobhani 